\title{
Neuroprotective effects of salidroside administration in a mouse model of Alzheimer's disease
}

\author{
QINGYUN LI $^{1 *}, \mathrm{JINHUA} \mathrm{WANG}^{2 *}, \mathrm{YUWANG} \mathrm{LI}^{1}$ and XIAOLIN XU ${ }^{1}$ \\ ${ }^{1}$ Department of Neurology, Tianjin Huanhu Hospital, Tianjin 300350; ${ }^{2}$ Department of Neurology, \\ Taizhou Central Hospital (Taizhou University Hospital), Taizhou, Zhejiang 318000, P.R. China
}

Received March 7, 2017; Accepted September 6, 2017

DOI: $10.3892 / \mathrm{mmr} .2018 .8757$

\begin{abstract}
Salidroside administration improves memory in different models of learning. However, its influence on models of Alzheimer's disease (AD) has not been widely studied. In the present study, the therapeutic effect of salidroside was investigated in an animal model of AD. APPswe/PS1 $1 \Delta 9$ mouse $(n=20)$ were randomly divided into either the AD model group or the salidroside $+A D$ model group $(n=10$ in each group), and C57BL/6J mouse $(n=20)$ of identical age and genetic background were randomly divided into either the normal control (NC) group or the salidroside $+\mathrm{NC}$ group ( $n=10$ in each group). The Morris water maze behavioral test was applied to all mice in order to investigate the effects of salidroside administration on learning and memory functions. The concentrations of malondialdehyde (MDA), glutathione (GSH) and nitrate in the hippocampus of the mice were determined, and hippocampal superoxide dismutase (SOD) activity was also determined. In addition, terminal deoxynucleotidyl-transferase-mediated dUTP nick end labeling was used to investigate the rate of neuronal apoptosis in the hippocampus. Furthermore, the concentrations of interleukin- 6 (IL-6) and tumor necrosis factor- $\alpha$ (TNF- $\alpha$ ) were tested for in the brain tissues of AD mice. Learning and memory functions in $\mathrm{AD}$ mice were revealed to improve following administration of salidroside. Furthermore, salidroside administration was revealed to decrease the concentrations of MDA and
\end{abstract}

Correspondence to: Dr Xiaolin Xu, Department of Neurology, Tianjin Huanhu Hospital, 6 Jizhao Road, Jinnan, Tianjin 300350, P.R. China

E-mail:xuxltj@163.com

Abbreviations: AD, Alzheimer's disease; MWM, Morris water maze; SOD, superoxide dismutase; GSH, glutathione; MDA, malondialdehyde; TNF- $\alpha$, tumor necrosis factor- $\alpha$; IL-10, interleukin-10

*Contributed equally

Key words: APPswe/PS1 $1 \mathrm{E} 9$ mouse, salidroside, SOD, MDA, GSH, nitrate, TNF- $\alpha$, IL-10 nitrate in the hippocampus, decrease the apoptotic rate of hippocampal neurons, and increase the activity of SOD and the concentration of GSH in hippocampal tissue. In addition, it was demonstrated that salidroside administration suppressed the expression levels of IL-6 and TNF- $\alpha$. In conclusion, this study revealed that the administration of salidroside could attenuate the effects of AD-associated memory and learning impairment in mice. Furthermore, it was demonstrated that the effects of salidroside administration on AD mice were, at least partially, via inhibition of brain oxidative/nitrosative damage, suppression of both IL- 6 and TNF- $\alpha$ expression levels, and suppression of the hippocampal neuronal apoptotic rate.

\section{Introduction}

Alzheimer's disease (AD) is a progressive neurodegenerative disorder, which is characterized by severe memory loss and behavioral disturbances (1-3). AD is a multifactorial disease and is affected by genetic risk factors, aging and oxidative stresses $(4,5)$. The impairment of memory and cognition in patients with AD is caused by synaptic loss, enhanced inflammatory signaling, progressive deposition of senile plaques, neurofibrillary tangles and neurodegeneration (6-8). Additionally, amyloid- $\beta$ (A $\beta)$ peptides, as well as the tau protein, in the form of neurofibrillary tangles, are implicated in the pathogenesis of $\mathrm{AD}$ (9). In addition to the tau-amyloid signature, oxidative damage, neuroinflammation, widespread synaptic loss and neuronal death are considered hallmark features of $\mathrm{AD}(9)$.

With an aging population, $\mathrm{AD}$ is on the increase, and there are currently no effective treatments or cures. Therefore, the pursuit of novel disease-modifying therapeutics for AD is the subject of intense investigation.

Salidroside is a type of traditional Chinese medicine, which is extracted from rhodiola medical plants, named Rhodiolasa chinensis A Bor. Salidroside has numerous pharmacological activities, such as anti-anoxia $(10)$, anticancer $(11,12)$, anti-fatigue (13), and anti-toxin effects (14). Furthermore, numerous studies have suggested that salidroside may also improve cognitive functioning in disease models $(15,16)$; however, the mechanisms were not determined. In this study, the potential therapeutic effect of salidroside in APPswe/PS1 1 E9 mice and its underlying mechanisms were investigated. 


\section{Materials and methods}

Experimental animals. All the experiments were performed with the approval of the Ethics Committee of the Tianjin Medical University (Tianjin, China). APPswe/PS1 $\triangle \mathrm{E} 9$ mice $(\mathrm{n}=20 ; 3$ months, male, 26-30 g) and C57BL/6J mice $(\mathrm{n}=20$; 3 months, male, 23-26 g) in clean grade were obtained from Beijing Huafukang Biological Technology company (Beijing, China) and raised in the temperature of $25 \pm 2^{\circ} \mathrm{C}$, relative humidity $70 \%$ and in a 12 -h light/dark room at with free access to food and water. Following 1 week of adaption, all APPswe/PS1 $\triangle \mathrm{E} 9$ mice were randomly divided into either the $\mathrm{AD}$ model group or the salidroside $+\mathrm{AD}$ model group $(\mathrm{n}=10$ in each group). C57BL/6J mice were also randomly divided into either the normal control (NC) group or the salidroside + NC group ( $\mathrm{n}=10$ in each group). The mice in the salidroside $+\mathrm{NC}$ and salidroside + AD model groups were administered salidroside $(30 \mathrm{mg} / \mathrm{kg}$ ) orally once daily for 3 consecutive months. Following treatment, behavioral tests and biochemical experiments were performed.

Morris water maze (MWM). Following the 3 months of salidroside treatment, the cognitive behavior of all of the mice was investigated using MWM (17), which measures spatial learning and memory ability. The animals were subjected to a daily session of four training trials for 4 consecutive days. In each training session, mice were placed into the pool at four different starting points (different quadrants). The mice were then permitted to find the platform within a maximum time period of $120 \mathrm{sec}$ and following that the mice were allowed to remain on the platform for a further $30 \mathrm{sec}$. If a mouse was unable to find the platform within the $120 \mathrm{sec}$ time period, the mouse would then be guided to the platform by the experimenter, and the escape latency was recorded as $120 \mathrm{sec}$. At day 5 of spatial testing, the platform was removed and mice were placed into water at any starting point. The time period that the mouse remained in the quadrant where the platform had previously been placed was then recorded.

Terminal deoxynucleotidyl-transferase-mediated dUTP nick end labeling (TUNEL) assay. Detection of hippocampal neuronal apoptosis was carried out using the TUNEL assay. The hippocampal tissue was fixed in $4 \%$ formalin at $4^{\circ} \mathrm{C}$ for $48 \mathrm{~h}$ and then embedded in paraffin wax. Apoptosis rates were detected using the In Situ Cell Death Detection kit (Roche Applied Science, Rotkreuz, Switzerland) according to the manufacturer's instructions. Apoptotic rate changes were measured via a light microscope (Olympus Corporation, Tokyo, Japan). Hippocampal tissue was fixed in $4 \%$ paraformaldehyde for $48 \mathrm{~h}$ at $4^{\circ} \mathrm{C}$ and embedded into paraffin wax. In Situ Cell Death Detection kit was used to detect the apoptosis according to the manufacturer's instructions. The images were taken using a light microscope (Olympus Corporation). Apoptotic rates were then determined according to the method previously detailed by Soslow et al (18). A positive expression rate of 0-1\% was defined as score 0 (negative), $1-10 \%$ as score 1 (weakly positive), $10-50 \%$ as score 2 (positive), $50-80 \%$ as score 3 (medium positive) and $80-100 \%$ as score 4 (strongly positive). Three sections were used for each group, and five fields were randomly selected from each section (magnification, x400).
Superoxide dismutase (SOD) and malondialdehyde (MDA) measurements. Following the MWM test, all of the mice were anaesthetized, immediately sacrificed, and brain tissues were quickly isolated. Following this, the brains were separated into two cerebral hemispheres via incision along the midline. Hippocampal tissue of CA1 region was then isolated from one of the cerebral hemispheres of each animal and then immediately frozen in liquid nitrogen for subsequent SOD and MDA testing. Hippocampal SOD (cat. no. ab65354) and MDA (cat. no. ab65354) (both from Abcam, Cambridge, UK) activity were then detected usinga microplate reader (wavelength, $210 \mathrm{~nm})$.

Nitrate assay. Colorimetric reaction using Griess reagent was used to investigate the nitrate concentration in the hippocampal tissue of the mouse. The Nitric Oxide Assay kit was purchased from Abcam, and all procedures were performed out in accordance with the manufacturer's instructions. The results of the hippocampal nitrate concentrations of the different groups were expressed as $\mu \mathrm{g} / \mathrm{mg}$ protein.

Determination of glutathione (GSH) concentration. Firstly, the hippocampal rat samples were precipitated by the addition of 5\% sulfosalicylic acid and protein-free supernatant was removed by centrifugation at $2,000 \mathrm{x} g$ for $10 \mathrm{~min}$. A sample of $100 \mu 1$ protein-free supernatant of the cell lysate was then added to $800 \mu \mathrm{l} 0.3 \mathrm{mM} \mathrm{Na}_{2} \mathrm{HPO}_{4}$ and $100 \mu \mathrm{l} 0.04 \%$ 5,5 '-dithiobis-2-nitrobenzoic acid (DTNB) in $0.1 \%$ sodium citrate at $4^{\circ} \mathrm{C}$ overnight. Following this, the absorbance of DTNB was monitored using a spectrophotometer at $412 \mathrm{~nm}$ for 5 min. A standard curve of GSH was established, and the sensitivity of measurement was determined to be between $1-100 \mu \mathrm{M}$. The results of the GSH concentrations in the samples were expressed as $\mu \mathrm{g} / \mathrm{mg}$ protein.

Determination of tumor necrosis factor- $\alpha(T N F-\alpha)$ and interleukin-6(IL-6) concentrations in tissue homogenate by ELISA. The concentrations of TNF- $\alpha$ and IL- 6 in the hippocampal tissue of the mice were determined using ELISA. ELISA kits for TNF- $\alpha$ (cat. no. ab46070) and IL-6 (cat. no. ab100772) were purchased from Abcam. All procedures were performed in accordance with the manufacturer's instructions. The results of the determined hippocampal concentrations of TNF- $\alpha$ and IL-6 were expressed as $\mu \mathrm{g} / \mathrm{mg}$ protein.

Statistical analysis. Statistical analysis was performed using SPSS 17.0 (SPSS, Inc., Chicago, IL, USA). All data were expressed as the mean \pm standard deviation of the mean. One-way analysis of variance was used to compare differences among three or more groups, and this was followed by Student Newman-Keuls-test for multiple comparisons. $\mathrm{P}<0.05$ was considered to indicate a statistically significant difference.

\section{Results}

Administration of salidroside improves the spatial learning and memory abilities of AD mice. MWM was used to investigate the effects of salidroside administration on the learning and memory abilities of mice. As revealed in Fig. 1A, the escape latency time of the AD group was longer than 

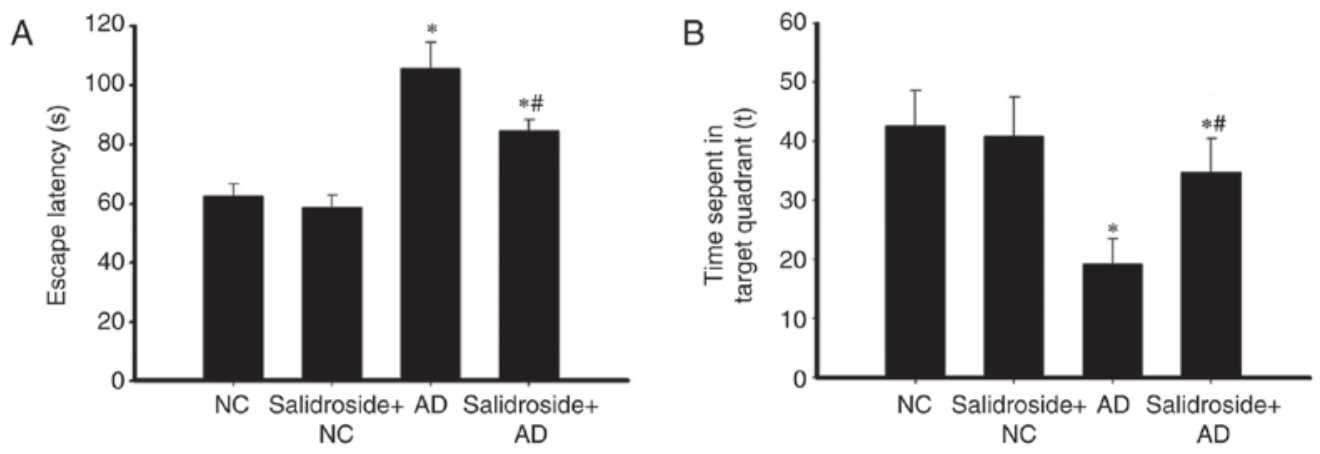

Figure 1. Salidroside administration improves the spatial learning and memory abilities of the AD animals ( $\mathrm{n}=10)$. Results are presented as (A) escape latency and (B) time spent in target quadrant. " $\mathrm{P}<0.05$ vs. normal control (NC) group; ${ }^{~} \mathrm{P}<0.05$ vs. AD group. AD, Alzheimer's disease; NC, normal control.

that of the normal group, and there was no significant difference between the normal and the salidroside $+\mathrm{NC}$ groups. Furthermore, the escape latency of the mice in the salidroside + AD model group was significantly reduced compared with the escape latency of the AD model group. As demonstrated in Fig. 1B, the time period that the AD model group spent in the target quadrant was significantly less than that spent by the normal group. Additionally, there was no significant difference in the time period spent in the target quadrant between the NC and the salidroside $+\mathrm{NC}$ groups. Furthermore, the time period that the mice of the salidroside + AD model group spent in the target quadrant was significantly higher than the time spent by the AD group, but significantly less than the time spent by the NC group. Results in the salidroside + AD group, the escape latency was significantly shortened, the time of swim in the platform quadrant significantly increased, suggesting that mice significantly improved learning and memory to escape the incubation period $(\mathrm{P}<0.05$; Fig. 1$)$.

Salidroside administration mitigates the rate of neuronal apoptosis in the CAl hippocampal region in AD mice. As revealed by Fig. 2, the number of neurons undergoing apoptosis in the CA1 hippocampal region was significantly increased in the AD group compared with the other groups. However, the administration of salidroside reduced this effect, as there was a significant reduction in neuronal apoptotic rates between the salidroside $+\mathrm{AD}$ group and the $\mathrm{AD}$ group.

Salidroside administration increases the concentration of $G S H$ and the activity of SOD in the hippocampus of AD mice. The activity of SOD and the concentration of GSH in hippocampal tissue were investigated. As demonstrated in Fig. 3A, the relative SOD activity was significantly decreased in the AD group compared with the NC group; whereas SOD activity was upregulated in the salidroside $+\mathrm{AD}$ group compared with the AD group. Furthermore, there was no significant difference between the SOD activity of the NC group and of the salidroside $+\mathrm{NC}$ group. Compared with the NC group, the GSH concentration was decreased in the AD group; whereas GSH concentration was upregulated in the salidroside $+A D$ group compared with the AD group. In addition, there was no significant difference between the GSH concentration of the $\mathrm{NC}$ group and the salidroside + NC group (Fig. 3B).
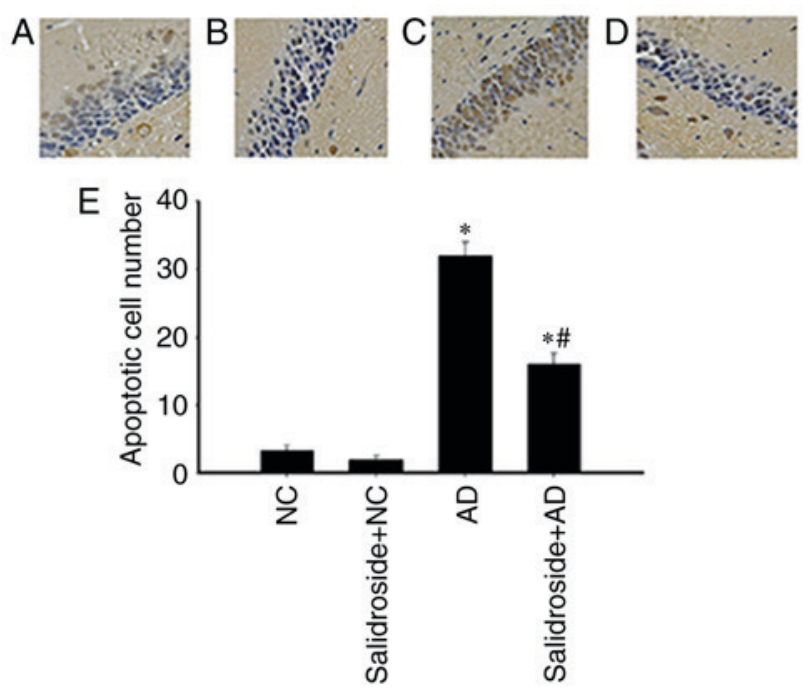

Figure 2. Salidroside administration mitigates the rate of neuronal apoptosis in the CA1 hippocampal region in AD mice. $n=5 /$ group. Three sections were used for each group, and five fields of view were randomly selected from each section to be viewed under a microscope. (A) NC group, (B) salidroside + NC group, (C) AD model, (D) salidroside + AD group. (E) Quantification data of the cellular apoptotic rate in different groups. ${ }^{*} \mathrm{P}<0.05$ vs. $\mathrm{NC}$ group; ${ }^{\#} \mathrm{P}<0.05$ vs. AD group. AD, Alzheimer's disease; NC, normal control.

Salidroside administration reduces the concentrations of $M D A$ and nitrate in the hippocampus of AD mice. The hippocampal concentrations of MDA and GSH in mice were also investigated. As revealed in Fig. 4, in comparison with the NC group, MDA and nitrate concentrations were both increased in the AD group; whereas both MDA and nitrate concentrations were downregulated in the salidroside $+\mathrm{AD}$ group compared with the AD group. Furthermore, there was no significant difference between the MDA and nitrate concentrations of the $\mathrm{NC}$ group and the salidroside $+\mathrm{NC}$ group.

Salidroside administration decreases the expression of TNF- $\alpha$ and IL-6 in the hippocampus of AD mice. The effects of salidroside administration with regards to induced inflammation were investigated. The expressions of TNF- $\alpha$ and IL-6 inflammatory factors decreased significantly in the hippocampus of the salidroside $+\mathrm{AD}$ group compared with mice belonging to the AD group (Fig. 5). There was no significant difference between the expression levels of TNF- $\alpha$ and IL- 6 of the NC group and the salidroside $+\mathrm{NC}$ group. 

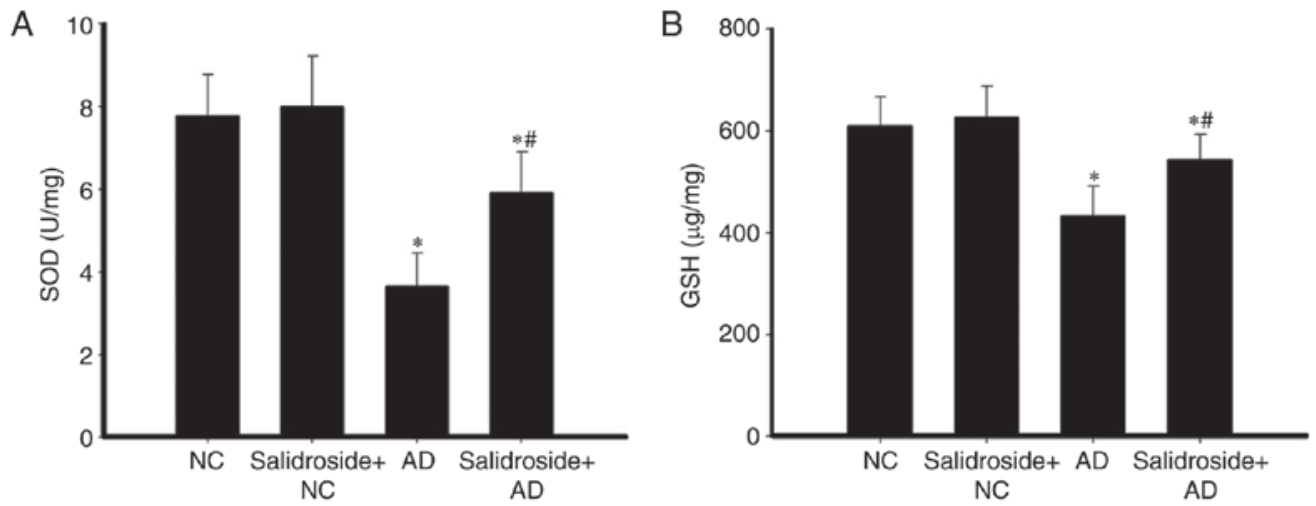

Figure 3. Hippocampal SOD activity and GSH concentration in the four different groups (mean \pm standard deviation, $n=5$ ). Results are displayed as (A) SOD activity, and (B) GSH concentration. Statistical analysis was performed using one-way analysis of variance with Student-Newman-Keuls test. "P $<0.05$ vs. NC group; ${ }^{\text {P }}<0.05$ vs. AD group. SOD, superoxide dismutase; GSH, glutathione; $\mathrm{NC}$, normal control; AD, Alzheimer's disease.
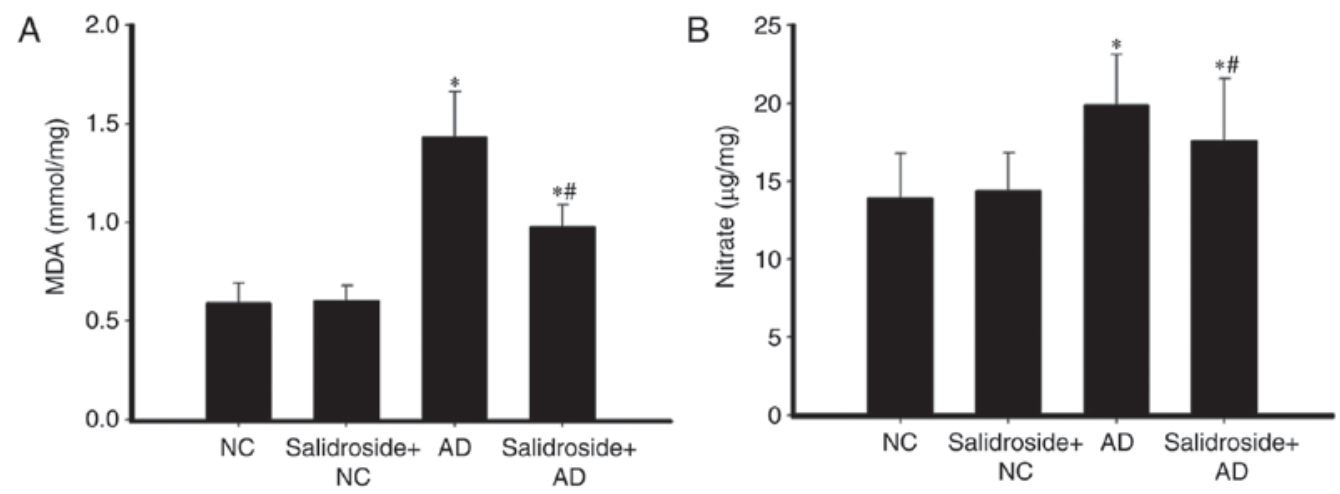

Figure 4. Hippocampal MDA and nitrate concentrations in the four different groups (mean \pm standard deviation; $\mathrm{n}=5$ ). Results are displayed as (A) MDA concentration, and (B) nitrate concentration. Statistical analysis was performed using one-way analysis of variance with Student-Newman-Keuls test. ${ }^{*} \mathrm{P}<0.05$ vs. NC group; ${ }^{\#} \mathrm{P}<0.05$ vs. AD group. MDA, malondialdehyde; $\mathrm{NC}$, normal control; AD, Alzheimer's disease.
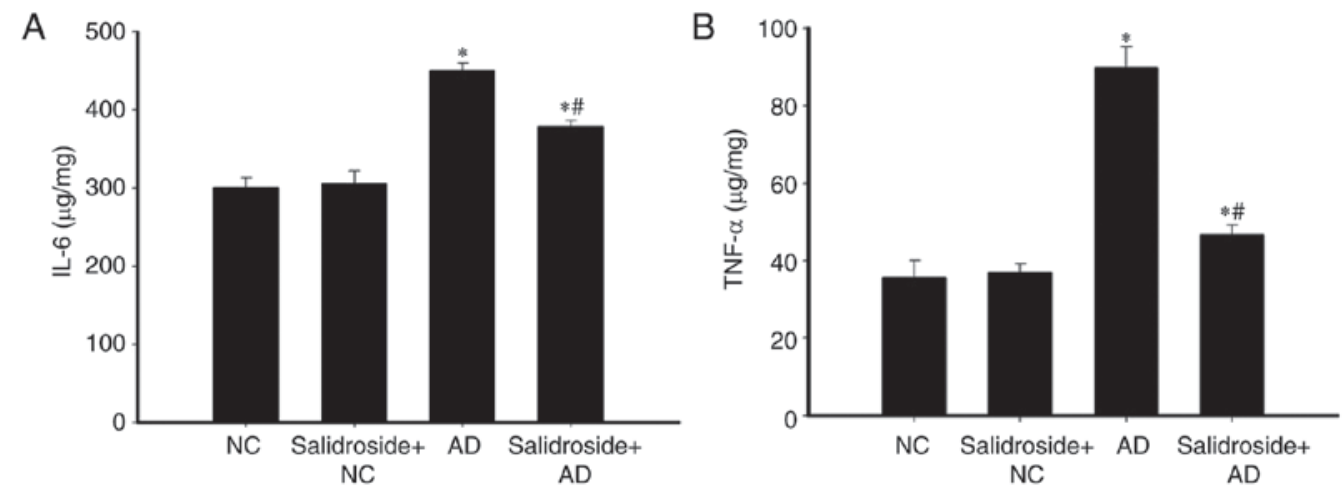

Figure 5. Hippocampal concentrations of IL- 6 and TNF- $\alpha$ in the four different groups (mean \pm standard deviation; $n=5$ ). Results are displayed as (A) concentration of IL-6, and (B) concentration of TNF- $\alpha$. Statistical analysis was performed using one-way analysis of variance with Student-Newman-Keuls test. ${ }^{*} \mathrm{P}<0.05$ vs. NC group, ${ }^{\#} \mathrm{P}<0.05$ vs. AD group. IL-6, interleukin-6; TNF- $\alpha$, tumor necrosis factor- $\alpha, \mathrm{NC}$, normal control; AD, Alzheimer's disease.

\section{Discussion}

The number of people with AD worldwide is increasing as a result of the aging population. Previous research has revealed that 46.8 million people worldwide suffer from dementia, and further research has indicated that this number may increase 2-3-fold by 2030 (19). AD is a severe neurodegenerative disease of the central nervous system, which results in learning and memory impairment. The rising rate of senile dementia is of serious concern to global healthcare systems and causes serious economic burdens to affected families and wider society. AD is a neurologically complex disorder, and thus it is likely that a combinatorial therapeutic approach will be needed for effective treatment.

Chinese herbology has been heavily used globally and is regarded as an importance source of medicines. Salidroside is a component of Rhodiolarosea, and possesses medicinal properties, such as anti-inflammatory $(20,21)$, antioxidative (14) 
and neuroprotective effects (22). In the present study, it was demonstrated that salidroside administration attenuates the memory impairment exhibited in the AD rat model.

Previous studies have reported that one of the major brain regions affected by $\mathrm{AD}$-induced neurogenesis is the hippocampus, which is a vital brain region for learning and memory (23). Hippocampal neuronal damage adversely affects neural plasticity and synaptic regulation, and thus is heavily implicated in the pathogenesis of AD (24). In the present study, it was revealed that the rate of hippocampal neuronal apoptosis was significantly increased in the rat AD model. Furthermore, it was demonstrated that the rate of apoptotic neurons in the CA1 hippocampal region was clearly increased in the AD group, and that administration of salidroside could attenuate this effect. In addition, the results of this study suggest that the administration of salidroside improves cognitive function in the AD model via suppression of the rate of apoptosis of hippocampal neurons.

It has previously been reported that salidroside, a potent antioxidant, ameliorates memory impairment via anti-oxidative damage activity. The decline of learning and memory function in rodents and humans has previously been linked to excessive oxidative stress, which may lead to neuronal death. There is a growing body of research suggesting that oxidative stress is implicated in AD pathogenesis, along with increased levels of lipid peroxidation, and oxidation of both DNA and protein in the brains of AD sufferers $(25,26)$. SOD is an important neural antioxidant enzyme that can remove excess free radicals (27). In addition, oxidative damage may also cause damage to components of the antioxidant system, such as MDA $(28,29)$. The present study demonstrated that oxidative damage downregulated the activity of SOD and increased MDA concentration in rat hippocampal tissue. Furthermore, it was revealed that in the AD rat model with memory deficit, these adverse effects were greatly attenuated when salidroside was administered.

IL-6 is a cytokine that is secreted by $\mathrm{T}$ cells and macrophages, and acts as both a pro-inflammatory and as an anti-inflammatory in order to stimulate an immune response (30,31). TNF- $\alpha$ can promote T cells to produce a variety of inflammatory factors, and therefore trigger inflammatory reactions $(32,33)$. In the present study, salidroside administration significantly decreased the expression of TNF- $\alpha$ and IL- 6 cytokine inflammatory factors.

In conclusion, the present study demonstrated that salidroside administration may improve cognitive function in AD mice. The protective effects maybe associated with alteration of the levels of free radicals in the hippocampus.

\section{Acknowledgements}

The present study was supported by a grant from Natural Science Foundation of Tianjin Municipal Science and Technology Commission (grant no. 13JCYBJC22700).

\section{References}

1. Diwu YC, Tian JZ and Shi J: Effects of Chinese herbal medicine Yinsiwei compound on spatial learning and memory ability and the ultrastructure of hippocampal neurons in a rat model of sporadic Alzheimer disease. Zhong Xi Yi Jie He Xue Bao 9: 209-215, 2011.
2. Riley KP, Snowdon DA and Markesbery WR: Alzheimer's neurofibrillary pathology and the spectrum of cognitive function: Findings from the num study. Ann Neurol 5: 567-577, 2002.

3. Gong CX, Liu F, Grundke-Iqbal I and Iqbal K: Postranslational modifications of tau protein in Alzheimer's disease. J Neural Transm 6: 813-838, 2005.

4. Moceri VM, Kukull WA, Emanual I, van Belle G, Starr JR Schellenberg GD, McCormick WC, Bowen JD, Teri L and Larson EB: Using census data and birth certificates to reconstruct the early-life socioeconomic environment and the relation to the development of Alzheimer's disease. Epidemiology 12: 383-389, 2001.

5. Iqbal K and Grundke-Iqbal I: Metabolic/signal transduction hypothesis of Alzheimer's disease and other tauopathies. Acta Neuropathol 109: 25-31, 2005.

6. Tuppo EE and Arias HR: The role of inflammation in Alzheimer's disease. Int J Biochem Cell Biol 37: 289-305, 2005.

7. Yin Y, Liu Y and Huang L: Anti-apoptosis effect of astragaloside Iv on Alzheimer's disease rat model via enhancing the expression of Bcl-2 and Bcl-Xl. Scandinavian J Lab Anim Sci 37: 75-82, 2010.

8. Chen Y, Tian Z, Liang Z, Sun S, Dai CL, Lee MH, LaFerla FM, Grundke-Iqbal I, Iqbal K, Liu F and Gong CX: Brain gene expression of a sporadic (icv-STZ Mouse) and a familial mouse model (3xTg-AD mouse) of Alzheimer's disease. PLoS One 7: e51432, 2012.

9. Javed H, Khan MM, Ahmad A, Vaibhav K, Ahmad ME, Khan A, Ashafaq M, Islam F, Siddiqui MS, Safhi MM and Islam F: Rutin prevents cognitive impairments by ameliorating oxidative stress and neuroinflammation in rat model of sporadic dementia of Alzheimer type. Neuroscience 210: 340-352, 2012.

10. Zhang J, Liu A, Hou R, Zhang J, Jia X, Jiang W and Chen J: Salidroside protects cardiomyocyte against hypoxia-induced death: A HIF-1 $\alpha$-activated and VEGF-mediated pathway. Eur J Pharmacol 607: 6-14, 2009.

11. Fang D, Chen Y, Xu B, Ren K, He ZY, He LL, Lei Y, Fan CM and Song XR: Development of lipid-shell and polymer core nanoparticles with water-soluble salidroside for anti-cancer therapy. Int J Mol Sci 15: 3373-3388, 2014.

12. Wang J, Li JZ, Lu AX, Zhang KF and Li BJ: Anticancer effect of salidroside on A549 lung cancer cells through inhibition of oxidative stress and phospho-p38 expression. Oncol Lett 7: 1159-1164, 2014.

13. Li M, Dong C, Huai L, Bende T, Lihua S and Ying W: Anti-fatigue effects of salidroside in mice. J Med Colleges PLA 23: 88-93, 2008.

14. Zhu Y, Shi Y, Wu D, Ji YJ, Wang X, Chen HL, Wu SS, Huang DJ and Jiang W: Salidroside protects against hydrogen peroxide-induced injury in cardiac $\mathrm{H} 9 \mathrm{c} 2$ cells via PI3K-Akt dependent pathway. DNA Cell Biol 30: 809-819, 2011.

15. ZovkoKoncic $M$ and Tomczyk $M$ : New insights into dietary supplements used in sport: Active substances pharmacological and side effects. Curr Drug Targets 14: 1079-1092, 2013.

16. Wang H, Ding Y, Zhou J, Sun X and Wang S: The in vitro and in vivo antiviral effects of salidroside from Rhodiolarosea $\mathrm{L}$. against coxsackie virus B3. Phytomedicine 16: 146-155, 2009.

17. Vorhees CV and Williams MT: Morris water maze: Procedures for assessing spatial and related forms of learning and memory. Nat Protoc 1: 848-858, 2006.

18. Soslow RA, Dannenberg AJ, Rush D, Woerner BM, Khan KN, Masferrer J and Koki AT: Cox-2 is expressed in human pulmonary, colonic and mammary tumors. Cancer 89: 2637-2645, 2000 .

19. Realdon O, Rossetto F, Nalin M, Baroni I, Cabinio M,Fioravanti R, Saibene FL, Alberoni M, Mantovani F, Romano M, et al: Technology-enhanced multi-domain at home continuum of care program with respect to usual care for people with cognitive impairment: The Ability-TelerehABILITation study protocol for a randomized controlled trial. BMC Psychiatry 16: $425,2016$.

20. Liu S, Yu X, Hu B, Zou Y, Li J, Bo L and Deng X: Salidroside rescued mice from experimental sepsis through anti-inflammatory and anti-apoptosis effects. J Surg Res 195: 277-283, 2015.

21. Díaz Lanza AM, Abad Martínez MJ, Fernández Matellano L, Recuero Carretero C, Villaescusa Castillo L, Silván Sen AM and Bermejo Benito P: Lignan and phenylpropanoid glycosides from Phillyrealatifolia and their in vitro anti-inflammatory activity. Planta Med 67: 219-223, 2001. 
22. Qu ZQ,Zhou Y,Zeng YS, Lin YK, Li Y,Zhong ZQ and Chan WY: Protective effects of a rhodiola crenulata extract and salidroside on hippocampal neurogenesis against streptozotocin-induced neural injury in the rat. PLoS One 7: e29641, 2012.

23. Kimura T, Hong Nguyen PT, Ho SA, Tran AH, Ono T and Nishijo H: T-817MA, a neurotrophic agent, ameliorates the deficits in adult neurogenesis and spatial memory in rats infused i.c.v. with amyloid- $\beta$ peptide. $\mathrm{Br} \mathrm{J}$ Pharmacol 157: 451-463, 2009

24. Palop JJ and Mucke L: Amyloid-beta-induced neuronal dysfunction in Alzheimer's disease: From synapses toward neural networks. Nat Neurosci 13: 812-818, 2010.

25. Arber CE, Li A, Houlden H and Wray S: Review: Insights into molecular mechanisms of disease in neurodegeneration with brain iron accumulation: Unifying theories. Neuropathol Appl Neurobiol 42: 220-241, 2016.

26. Zhao Y and Zhao B: Oxidative stress and the pathogenesis of alzheimer's disease. Oxid Med Cell Longev 2013: 316523, 2013.

27. Pencea V, Bingaman KD, Wiegand SJ and Luskin MB: Infusion of brain-derived neurotrophic factor into the lateral ventricle of the adult rat leads to new neurons in the parenchyma of the striatum, septum, thalamus and hypothalamus. J Neurosci 21: 6706-6717, 2001
28. Kudin AP, Bimpong-Buta NY, Vielhaber S, Elger CE and Kunz WS: Characterization of superoxide-producing sites in isolated brain mitochondria. J Biol Chem 279: 4127-4135, 2004.

29. Calvert JW, Yin W, Patel M, Badr A, Mychaskiw G, Parent AD and Zhang JH: Hyperbaric oxygenation prevented brain injury induced by hypoxia-ischemia in a neonatal rat model. Brain Res 951: $1-8,2002$.

30. Ferguson-Smith AC, Chen YF, Newman MS, May LT, Sehgal PB and Ruddle FH: Regional localization of the interferon-beta $2 / \mathrm{B}$-cell stimulatory factor $2 /$ hepatocyte stimulating factor gene to human chromosome 7p15-p21. Genomics 2: 203-208, 1988.

31. van der Poll T, Keogh CV, Guirao X, Buurman WA, Kopf M and Lowry SF: Interleukin-6 gene-deficient mice show impaired defense against pneumococcal pneumonia. J Infect Dis 176: 439-444, 1997.

32. Hotamisligil GS, Shargill NS and Spiegelman BM: Adipose expression of tumor necrosis factor-alpha: Direct role in obesity-linked insulin resistance. Science 259: 87-91, 1993.

33. Tateya S, Kim F and Tamori Y: Recent advances in obesity induced inflammation and insulin resistance. Frontiers Endocrinol (Lausanne) 4: 93, 2013. 